\title{
Recall to a general practitioner or to a nurse clinic improved assessment in patients with coronary artery disease
}

\author{
Moher M, Yudkin P, Wright L, et al, for the Assessment of Implementation Strategies (ASSIST) Trial Collaborative Group. \\ Cluster randomised controlled trial to compare three methods of promoting secondary prevention of coronary heart \\ disease in primary care. BMJ 2001 Jun 2;322:1338-42.
}

QUESTION: In patients with coronary artery disease (CAD), what is the effectiveness of audit and feedback recall to a general practitioner (GP), and recall to a nurse clinic for improving secondary preventive care?

Source of funding: NHS Executive.

For correspondence: Dr M Moher, Department of Primary Health Care, University of Oxford, Institute of Health Sciences, Oxford OX3 $7 L F, U K$. Fax +44 (0) 1865226720

\section{Design}

Cluster randomised \{allocation concealed*\}†, un-

\section{Setting}

21 general practices in Warwickshire, UK. blinded,* controlled trial with 18 months of follow up.

Recall to a general practitioner (GP) or to a nurse clinic (NC) v audit and feedback (AF) for secondary preventive care of coronary artery disease

\begin{tabular}{|c|c|c|c|c|}
\hline \multirow[b]{2}{*}{ Outcomes at 18 months } & \multicolumn{3}{|c|}{ Mean percentage (baseline) } & \multirow{2}{*}{$\begin{array}{l}\text { Absolute difference } \\
\text { after adjusting for } \\
\text { baseline }(95 \% \mathrm{Cl})\end{array}$} \\
\hline & GP & NC & AF & \\
\hline \multirow[t]{2}{*}{ Adequate assessment } & $76(31)$ & - & $52(29)$ & $23 \%(10$ to 36$)$ \\
\hline & - & $85(29)$ & $52(29)$ & $33 \%(19$ to 46$)$ \\
\hline \multirow[t]{2}{*}{ Treatment with antiplatelet drugs } & $80(73)$ & - & $74(62)$ & $2 \%(-6$ to 10$) \ddagger$ \\
\hline & - & $85(66)$ & $74(62)$ & $10 \%(3$ to 17$)$ \\
\hline
\end{tabular}

‡ Not significant.

\section{COMMENTARY}

Moher $e t$ al investigated the "care gap" between optimal treatment and reality in managing patients with documented CAD. Participating practices (only 21 of 79 invited) were probably atypical of most British practices, and baseline standards of care were probably above average. Furthermore, the control group benefited from participation, and incremental costs of interventions were not described. Given these limitations, the study results can probably be generalised with caution.

Follow up and assessment by nurses were superior to follow up and assessment by physicians. However, prescribing in the active intervention groups was unchanged, and surrogate clinical outcomes were not improved. Adequacy of prescribing effective treatment seems to be a limiting step in optimising care. The authors partially rationalise the prescribing barrier as a consequence of negative physician and patient attitudes toward polypharmacy. Although such an attitude may affect prescribing, patients, not their physicians, suffer when effective treatments are withheld.

If patient, physician, or health system barriers to implementing effective treatments are to be overcome, radical and systematic changes rather than incremental, practitioner-dependent modifications may have to be introduced. A better understanding of factors that enable, motivate, and reward patients and health professionals for optimal behaviours is vital to considering healthcare innovations that might lead to improved assessment, prescribing, and clinical outcomes. If research findings are supportive, legal and professional regulatory changes will have to be considered.

Given the preeminent role of cardiovascular and cerebrovascular disease as a worldwide cause of mortality and a drain on healthcare resources, advances in the application of proven secondary prevention measures can have a substantial payoff for older, individual patients and for society in general.

S George Carruthers, MD United Arab Emirates University Al Ain, United Arab Emirates
Patients

2142 patients with established CAD (a previous diagnosis of myocardial infarction and angina, and receiving antianginal drugs or revascularisation by percutaneous transluminal coronary angioplasty or coronary artery bypass). 1906 patients (mean age 66 y, 68\% men) completed the study.

\section{Intervention}

7 practices were allocated to 1 of 3 quality improvement approaches: audit and feedback (audit group, 559 patients), recall to the GP (GP recall group, 682 patients), and recall to the nurse clinic (nurse recall group, 665 patients). Summary audit results of preventive care were given to each practice at baseline. Practices assigned to the GP and nurse recall groups were given resources to set up registers and recall systems for regular review of patients.

\section{Main outcome measures}

The primary outcome was adequate assessment of 3 risk factors (blood pressure, cholesterol, and smoking status) at 18 months. The main secondary outcomes were treatment with hypotensive agents, lipid lowering drugs, and antiplatelet drugs.

\section{Main results}

The increase in adequately assessed patients was greater in the GP and nurse recall groups than in the audit group ( $p \leqslant 0.002)$ (table). The increase in use of antiplatelet drugs was greater in the nurse recall group than in the audit group $(\mathrm{p}=0.009)$, but the GP recall and audit groups did not differ for use of antiplatelet drugs $(p=0.61)$ (table). The groups did not differ for change from baseline of the recorded treatment with hypotensive agents (rates at follow up were 70\%, 73\%, and 66\% for the audit, GP recall, and nurse recall groups, respectively) $(\mathrm{p}=0.35)$ or lipid lowering drugs (rates at follow up were $37 \%, 41 \%$, and $40 \%$ for the audit, GP recall, and nurse recall groups, respectively) $(\mathrm{p}=0.63)$. Furthermore, the groups did not differ for clinical outcomes (blood pressure, total cholesterol concentrations, or cotinine concentrations; all $\mathrm{p}$ values $>0.05$ ).

\section{Conclusion}

In patients with coronary artery disease, recall to a general practitioner or to a nurse clinic was more effective than audit and feedback for improving risk assessment but not for drug prescribing or clinical outcomes.

*See glossary.

†Information provided by author. 